\title{
Effects of ambient turbulence and fuel properties on the evaporation rate of single droplets
}

\author{
J.-S. Wu ${ }^{\mathrm{a}, *}$, Y.-J. Liu ${ }^{\mathrm{b}}$, H.-J. Sheen ${ }^{\mathrm{b}}$ \\ a Department of Mechanical Engineering, National Chiao-Tung University, 1001 Ta-Hsueh Road, Hsinchu 30050, Taiwan \\ ${ }^{\mathrm{b}}$ Institute of Applied Mechanics, National Taiwan University, Taipei 10670, Taiwan
}

Received 16 October 2000; received in revised form 16 March 2001

\begin{abstract}
Results of the evaporation of a single liquid fuel droplet in various free-stream turbulence intensities and scales are reported. Experiments are carried out at room temperature by using $n$-heptane and $n$-decane fuels at $R e_{d}=100$. A lowspeed vertical wind tunnel with different turbulence intensities and scales, controlled by using different sizes of disk, is constructed. The free-stream turbulence intensities are varied in the range from $1 \%$ to $60 \%$ and the integral length scales are from 2.5 to 20 times of the initial droplet diameter. Results show that the time history of droplet diameter follows the $d^{2}$-law in turbulent environments with generally higher evaporation rates as compared with those in quasi-laminar cases. Combined effects of liquid fuel properties and ambient turbulence properties on the evaporation rate can be reasonably well explained by the correlation of normalized evaporation rate with the effective vaporization Damköhler number, $D a_{\mathrm{v}}$. (C) 2001 Elsevier Science Ltd. All rights reserved.
\end{abstract}

\section{Introduction}

In practical industrial and military applications, liquid fuels are often used in combustion systems. Normally the liquid fuel is atomized into small droplets in combustion chamber to increase the contact area between fuel liquid and air (or to speed up the evaporation rate). In general, three most important factors affecting the combustion efficiency are temperature, ambient turbulence and fuel residence time. These are so-called 3-T factors in combustion chamber design process. The magnitude of fuel residence time decides the size of the combustion chamber. Those variables, which influence the fuel residence time, include ambient temperature and pressure, size of droplets, velocity distribution, ambient turbulence and liquid fuel properties. Therefore, it is important to study the effects of these conditions on the liquid droplet evaporation.

Because of the importance of fundamentals and applications to spray combustion, liquid droplet evapora-

\footnotetext{
${ }^{*}$ Corresponding author. Tel.: +886-3-573-1693; fax: +886-3572-0634.

E-mail address: chongsin@cc.nctu.edu.tw (J.-S. Wu).
}

tion has been studied intensively in the past, for detailed reviews see [1,2], and references cited therein. In the practical spray system, the liquid droplet evaporation phenomenon is very complicated, comprising the liquid phase, the fuel vapor phase and the surrounding forced convection with turbulence, and the interactions among each other. Not only the understanding of liquid droplet evaporation is crucial in essence, it also plays an important role in determining the ignition time delay and ignition location of droplets. Most research simplified the sprays into the clouds of monodispersed (or polydispersed) liquid droplets. Hence, the detailed understanding of the evaporation process and the mechanism of a single liquid droplet becomes the first important step in modeling and simulating the practically complicated spray system.

There were numerous researches conducted in the past attempting to study the evaporating process of a single liquid droplet, including experimental and numerical studies; e.g., the references cited in $[1,2,5]$. Most experimental studies were carried out for a single liquid droplet in natural convection or in laminar forced convection. Very few researchers have studied the effects of ambient turbulence on the droplet vaporization in forced convection condition. Additionally, most 


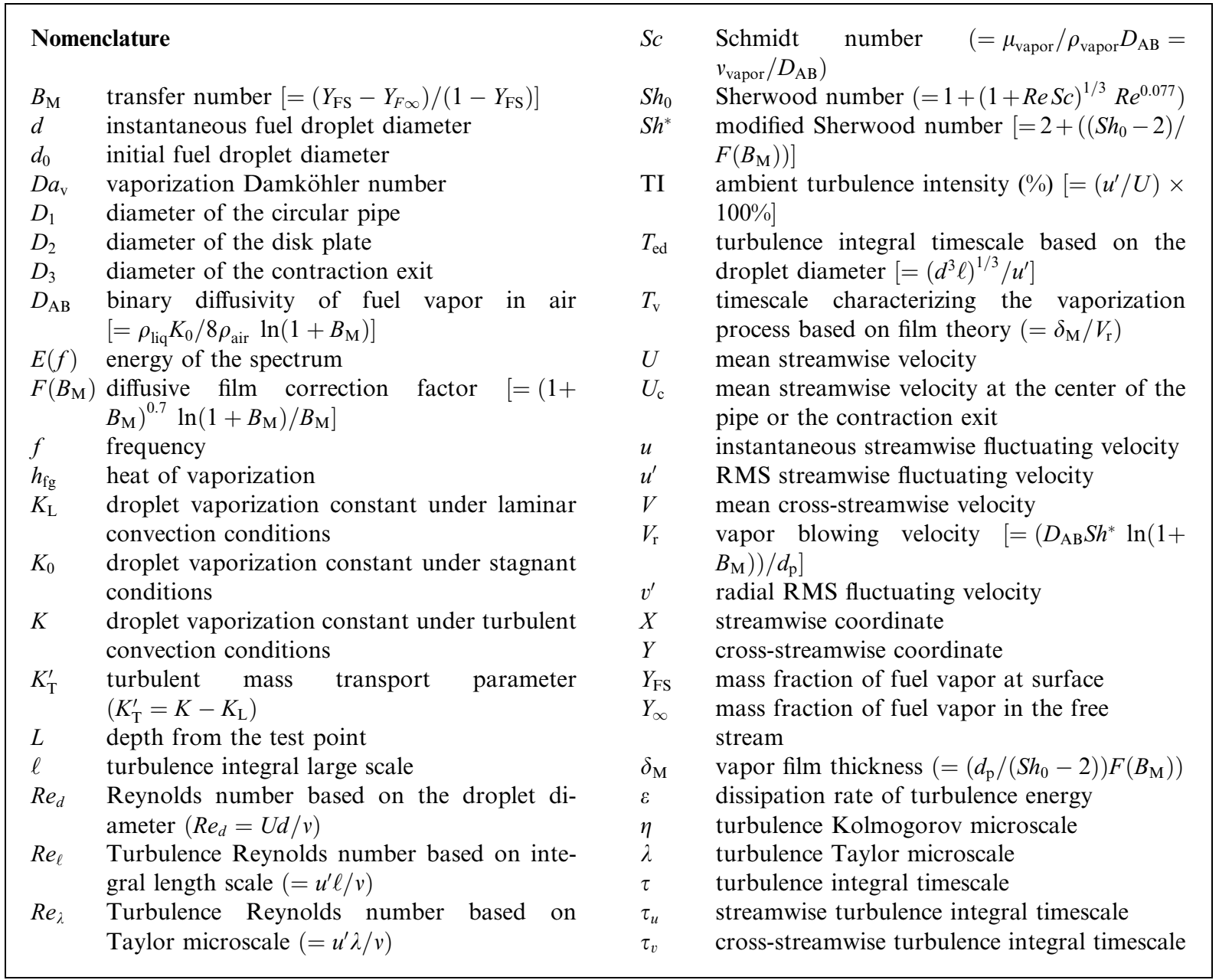

numerical studies have assumed no free-stream turbulence effects on the droplet evaporation rates, since the size of large eddy in the free-stream turbulence is often much larger than the droplet diameter, indicating the local laminar flow exists from the viewpoints of the droplet [3]. However, free-stream turbulence is composed of continuous spectrum of various length scales and timescales. Therefore, there exist other smaller length scales and timescales in the turbulence that may greatly affect the evaporation rate of the liquid droplets. In order to better understand the liquid droplet evaporation, it is necessary to clarify the liquid-gas inter-phase transport by studying the interaction between different length scales of ambient turbulence and liquid droplet [4].

Recently there have been few experimental reports studying the effects of ambient turbulence on the evaporation rate of a single liquid droplet in forced convection [5-9]. They are briefly reviewed in the following in turn.
Gokalp et al. [5] measured the evaporation rates of single $n$-heptane and $n$-decane liquid droplets in an unheated turbulent environment. The turbulence intensities were up to $44 \%$ and the integral length scales were much larger than the initial size of the liquid droplet $(\ell / d \geqslant 5)$. Their results showed that the ambient turbulence had little influence on the evaporation rate of $n$ heptane droplet (more volatile); however, it had strong influence on that of $n$-decane droplet (less volatile). They further found that $K_{\mathrm{T}}^{\prime}\left(=K_{\mathrm{T}}-K_{\mathrm{L}}\right)$ of $n$-decane droplet increases more than $50 \%$ in some cases. In addition, the authors proposed an effective vaporization Damköhler number, $D a_{\mathrm{v}}=T_{\text {ed }} / T_{\mathrm{v}}$, where $T_{\text {ed }}$ is the turbulence eddy timescale based on the initial droplet size and $T_{\mathrm{v}}$ is the vaporization timescale based on the film theory, to explain the combined effects of fuel and ambient turbulence properties on droplet evaporation. However, a systematic study revealing the correlation between $D a_{\mathrm{v}}$ and droplet evaporation rate seems still not available. Moreover, the size of liquid droplet also influences the 
evaporation rate. It was concluded that ambient turbulence effect is more pronounced as the droplet becomes smaller.

Hiromitsu and Kawaguchi [6] measured the evaporation rates of different paraffin hydrocarbons, distilled water and ethanol in the forced convection with various temperatures (323-423 K) and turbulence intensities (3$20 \%$ ). They found that the free-stream turbulence has strong influence on the evaporation rate of single liquid droplets. Furthermore, they pointed out that the use of effective vaporization Damköhler number by Gokalp et al. [5] is erroneous without consideration of temperature effects. However, it is problematic to use the droplet surface temperatures obtained in stagnant condition to compute the effective Damköhler number as conducted in [6]. Therefore, the temperature effect on the effective Damköhler number is still an open question and requires further studies.

Yearling [7] studied the heat and mass transfer rates of single spherical water, ethanol, or methanol droplets in heated air flows with controlled ambient turbulence. $\mathrm{He}$ concluded that even a mild gas-phase turbulence (about 5-10\%) could lead to an increase on the heat and mass transfer rates of the liquid droplets by as much as $30-50 \%$ when compared to those in laminar flow at the same Reynolds number.

Later in the same group as Gokalp et al., Birouk et al. [8] developed a closed chamber, which could produce a quasi-zero mean velocity and nearly homogeneous and isotropic flow region. The chamber was used to study the effects of turbulence (without mean motion) on droplet vaporization with turbulence integral length scales much larger than the initial droplet diameter. The results showed that the presence of turbulence increased the average evaporation rates as compared to that of the stagnant case. The linear regression rate of droplet surface area versus time is observed for all the cases considered. Similar to Gokalp et al. [5], various fuels investigated respond differently to the same turbulent environment. For a given turbulent kinetic energy, ambient turbulence enhances most effectively the average vaporization rates of the lowest volatility fuels. A phenomenological model for the turbulence effects on monocomponent droplet vaporization was proposed and discussed in terms of the ratio between the turbulence diffusivity and the molecular diffusivity of the fuel vapor.

Very recently, Gokalp et al. [9] have further carried out the experiments, using the same test facility as in [8], on droplet breakup and vaporization relevant to highpressure spray combustion applications. Results showed that the droplet evaporation rates depend strongly on the reduced pressures and temperatures. Further, the evaporation rates essentially increased with the pressure when the reduced temperature was above unity. Droplet evaporation rates also depend strongly on turbulence and are proportional to $3 / 2$ power of the turbulence Reynolds number.

Past studies raises a need of systematic study of the combined effects of turbulence and fuel properties on the droplet vaporization. For example, is it possible to correlate the combined effects on droplet vaporization in terms of the effective vaporization Damköhler number, which was proposed by Goklap et al. [5]? Therefore, the present experimental investigation was undertaken to study these effects at room temperature (to remove the temperature effect at this moment) and reevaluate the validity of the vaporization Damköhler number in describing the droplet evaporation. The specific objectives of the current study are:

1. to characterize the ambient mean velocities and turbulence properties at the exit of a vertical open wind tunnel,

2. to measure the evaporation rates of single liquid droplets for different fuels in turbulent environments, and

3. to interpret the measurements using vaporization Damköhler number and to explain the combined effects of ambient turbulence and fuel properties on droplet vaporization at room temperature.

The paper begins with descriptions of experimental methods. Results are then considered treating ambient mean and turbulent properties, droplet vaporization, and correlation using vaporization Damköhler number in turn.

\section{Experimental methods}

\subsection{Apparatus}

The apparatus included an up-flowing, turbulent air wind tunnel, with the test liquid droplet suspended near the axis at the downstream end of the wind tunnel. The schematic diagram of the wind tunnel is shown in Fig. 1. A variable speed blower produced the turbulent airflow, followed by a temperature-controlled electric heater in the pipe, a honeycomb, a 1.3-m horizontal settling chamber (pipe), a $90^{\circ}$ smooth bend pipe, a honeycomb, a 15-to-1 (length-to-diameter ratio) long pipe (120 mm diameter) and a removable, a round-shaped contraction nozzle (16-to-1 in area ratio; $30 \mathrm{~mm}$ in diameter at the exit) at the end to provide a non-swirling uniform flow at the exit. As the contraction nozzle is removed, the exit flow properties are expected to be typical of a fully developed turbulent pipe flow. From the preliminary tests, the airflow could be heated up to $500 \mathrm{~K}$ and $0.5-10 \mathrm{~m} / \mathrm{s}$ at the tunnel exit. All hot portions of the wind tunnel were covered by heat-insulating material to decrease the disturbances caused by natural convection. This configuration provided both quasi-laminar (with the contraction nozzle) and typical turbulent (without the 


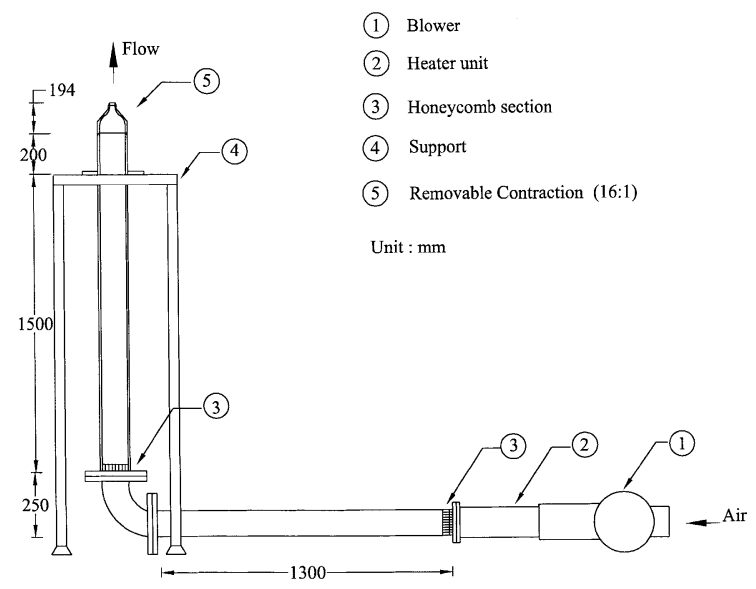

Fig. 1. Schematic diagram of wind tunnel for droplet evaporation.

contraction nozzle) pipe flow properties for further droplet evaporation experiments.

To generate the required turbulent intensities and length scales, a disk plate was placed at different locations within the vertical long pipe (Fig. 2), which similar technique has been used by Wu and Faeth [10]. By

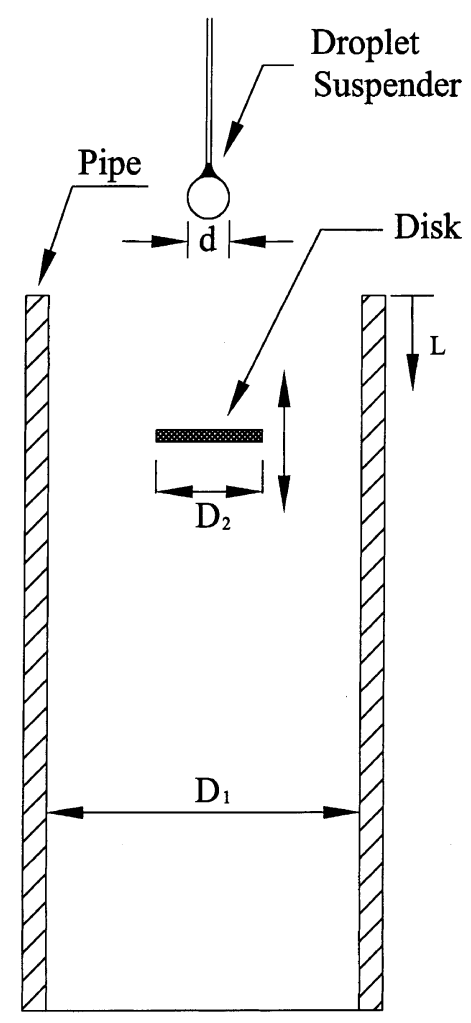

Fig. 2. Schematic diagram of turbulence generator for droplet evaporation. changing the disk sizes ( 20 and $60 \mathrm{~mm}$ ) and the distances from the exit $(80-650 \mathrm{~mm})$, turbulent flow properties at the test section (tunnel exit) could be varied. In general, turbulent environments with $u^{\prime} / U=1-60 \%$ and $\ell=3.6-38 \mathrm{~mm}$ were achieved at the test section with this arrangement.

Moreover, a special droplet suspender was fabricated to support the testing single liquid droplet (top of Fig. 2). It was made of a $100-\mu \mathrm{m}$ diameter quartz fiber with an enlarged spherical-shape tip, which was made of Epoxy adhesives. This design not only helped increase the contact surface area between liquid droplet and suspender, but also helped stabilize the droplet in the turbulent environments from our experience. For the current study, the liquid droplet diameters were either 1.5 or $2.5 \mathrm{~mm}$, depending on the flow mean velocity, to fix the Reynolds number at 100 . It was believed that the suspender would not affect the droplet vaporization much since the internal flow inside the liquid droplet would not be disturbed as in the previous studies, e.g., [5-9]. A pneumatic driven flowshielding aluminum plate, triggered by an electromagnetic valve, was installed between the suspended droplet and the tunnel exit. It was used to block the up-flowing air in order to prevent the droplet preevaporation before the experiment started. Test liquid droplet was generated using a syringe with $0.5 \mathrm{~mm}$ in tip diameter.

\subsection{Instrumentation}

Ambient flow structures in the test section were measured using a traverible $(5 \mu \mathrm{m}$ accuracy in positioning) laser velocimeter, based on a $514.5 \mathrm{~nm}$ line aircooled $300 \mathrm{~mW}$ argon-ion laser. The sending optics included a 2.27:1 beam expander yielding a measuring volume that has a diameter of $50 \mu \mathrm{m}$ and a length of 500 $\mu \mathrm{m}$. The flow was seeded with $\mathrm{MgO}$ seeding particle $(0.24 \mu \mathrm{m}$ nominal diameter, CERAC) at the inlet of the blower to yield data rates in the range of $0.5-5 \mathrm{kHz}$, depending on the mean velocity of the airflow. Velocity information were obtained from the output of a lowpass filtered counter (IFA 750, TSI). Data were collected using a DMA interface card (12 bit, TSI 6261 DMA, TSI), which was connected to a personal computer for further data analysis. Sampling rate was chosen less than half of the data rate to control alias signal. Further, all the experimental settings were made through a windowbased software, FIND, developed by TSI. Mean and fluctuating velocities were obtained using sampling periods of $120 \mathrm{~s}$, whereas temporal power spectrum were obtained by averaging at least 10 sets of data with similar duration. Experimental uncertainties (95\% confidence) largely were controlled by sampling limitations to yield the following values for mean velocities of 0.5-3 $\mathrm{m} / \mathrm{s}$ : mean velocities less than $5 \%$, fluctuating velocities 
less than $10 \%$, and temporal power spectrum less than $20 \%$. All measurements were repeatable within these ranges over a period of several months.

A digital CCD camera (TSI) with $1008 \times 1018$ pixels and 30 frames per second was used to record the droplet images. Image data were then downloaded to a personal computer right after the recording process for further image analysis. As mentioned earlier, shadowgraph was used to increase the signal-to-noise ratio of the image. In addition, a micro-Nikon $200 \mathrm{~mm}(f / 4)$ lens with extension ring was used to increase the resolution for image analysis. Diameter of liquid droplet was determined using equivalent spherical to ellipsoidal volume. Data were obtained by averaging over 3-5 times of experiments to reduce the experimental uncertainties. Estimated experimental uncertainties (95\% confidence) for droplet diameter was less than $3 \%$. All data were repeatable within this range over a period of several months.

\subsection{Test conditions}

Typical test conditions for suspended liquid droplets in turbulent environments with $\ell / d \approx 5$ and $u^{\prime} / U_{\mathrm{c}} \approx 10 \%$ are summarized in Tables 1 and 2 , respectively $\left(R e_{d}=100\right)$. Mean streamwise velocities at the axis of the tunnel were either 0.6 or $1.0 \mathrm{~m} / \mathrm{s}$ depending on the sizes of the droplet, 2.5 or $1.5 \mathrm{~mm}$, respectively. The ambient flow properties summarized in Tables 1 and 2 were averaged over the central region of the tunnel exit, e.g., $0.1 \leqslant y / D_{1} \leqslant 0.1$. In general, pipe flow properties without disk plate agreed with existing measurements in the literature within experimental uncertainties, though the current pipe Reynolds numbers were in the range of $4800-8000$, which was lower as compared with previous experiments [11-13]. Resulting isotropic values in fluctuating velocity, $u^{\prime} / v^{\prime}$, were in the range of $0.98-1.05$, which represented that the isotropic turbulence approximation was valid. Streamwise integral length scales were found using Taylor's hypothesis, $\ell=U \tau_{u}$, where $\tau_{u}$ was obtained from the measurements of temporal power spectrum of velocity fluctuations [14]. Resulting ratios of streamwise integral length scale to droplet diameter were in the range $2.5-20$, which was typical of dispersed phases in turbulent multiphase flows [15]. Komogorov scales were computed to be $\eta=\ell /\left(u^{\prime} \ell / v\right)^{3 / 4}$ from [16]. The resulting values of $\eta / d$ were in the range of $0.01-0.1$, which was generally much less than unity. Moreover, the turbulence Reynolds numbers, $R e_{\ell}$, ranged 16-280, which were definitely representative of fully developed turbulent flows as discussed by Tennekes and Lumley [16]. Two liquid fuels, $n$-heptane and $n$-decane, were used as the test droplets.

Table 1

Typical test conditions for droplet evaporation measurement $\left(\ell / d \approx 5, \operatorname{Re}_{d}=100\right)$

\begin{tabular}{|c|c|c|c|c|c|c|c|c|c|c|}
\hline Flow condition & $U_{\mathrm{c}}(\mathrm{m} / \mathrm{s})$ & $d(\mathrm{~mm})$ & $\begin{array}{l}u^{\prime} / U_{\mathrm{c}} \\
(\%)\end{array}$ & $\begin{array}{l}v^{\prime} / U_{\mathrm{c}} \\
(\%)\end{array}$ & $\begin{array}{l}u^{\prime} / v^{\prime} \\
(\%)\end{array}$ & $\ell / d$ & $\lambda / d$ & $\eta / d$ & $\operatorname{Re}_{\ell}$ & $R e_{\lambda}$ \\
\hline Pipe flow & 0.6 & 2.5 & 4.17 & 4.13 & 1.01 & 5.1 & 0.49 & 0.12 & 21.3 & 2.0 \\
\hline $\begin{array}{l}D_{2}=20 \mathrm{~mm} \\
L / D_{2}=12.5\end{array}$ & 0.6 & 2.5 & 10.0 & 9.7 & 1.03 & 4.4 & 0.25 & 0.05 & 43.8 & 2.5 \\
\hline $\begin{array}{l}D_{2}=60 \mathrm{~mm} \\
L / D_{2}=4.12\end{array}$ & 0.6 & 2.5 & 15.2 & 14.8 & 1.03 & 4.6 & 0.21 & 0.04 & 69.2 & 3.2 \\
\hline $\begin{array}{l}D_{2}=20 \mathrm{~mm} \\
L / D_{2}=2.5\end{array}$ & 1.0 & 1.5 & 33.5 & 32.8 & 1.02 & 5.4 & 0.15 & 0.02 & 181.7 & 5.0 \\
\hline $\begin{array}{l}D_{2}=60 \mathrm{~mm} \\
L / D_{2}=1.67\end{array}$ & 1.0 & 1.5 & 55.5 & 55.3 & 1.04 & 4.8 & 0.11 & 0.01 & 277.7 & 6.4 \\
\hline
\end{tabular}

Table 2

Typical test conditions for droplet evaporation measurement $\left(u^{\prime} / U_{\mathrm{c}} \approx 10 \%, \operatorname{Re}_{d}=100\right)$

\begin{tabular}{|c|c|c|c|c|c|c|c|c|c|c|}
\hline Flow condition & $U_{\mathrm{c}}(\mathrm{m} / \mathrm{s})$ & $d(\mathrm{~mm})$ & $\begin{array}{l}u^{\prime} / U_{\mathrm{c}} \\
(\%)\end{array}$ & $\begin{array}{l}v^{\prime} / U_{\mathrm{c}} \\
(\%)\end{array}$ & $\begin{array}{l}u^{\prime} / v^{\prime} \\
(\%)\end{array}$ & $\ell / d$ & $\lambda / d$ & $\eta / d$ & $R e_{\ell}$ & $R e_{\lambda}$ \\
\hline $\begin{array}{l}D_{2}=20 \mathrm{~mm} \\
L / D_{2}=10\end{array}$ & 0.6 & 2.5 & 10.2 & 9.7 & 1.05 & 3.8 & 0.23 & 0.04 & 38.4 & 2.3 \\
\hline $\begin{array}{l}D_{2}=60 \mathrm{~mm} \\
L / D_{2}=7.5\end{array}$ & 0.6 & 2.5 & 10.7 & 10.5 & 1.02 & 7.1 & 0.32 & 0.05 & 74.5 & 3.3 \\
\hline $\begin{array}{l}D_{2}=60 \mathrm{~mm} \\
L / D_{2}=8.33\end{array}$ & 1.0 & 1.5 & 10.1 & 9.9 & 1.02 & 12.0 & 0.42 & 0.06 & 121.3 & 4.2 \\
\hline $\begin{array}{l}D_{2}=60 \mathrm{~mm} \\
L / D_{2}=10.8\end{array}$ & 1.0 & 1.5 & 8.9 & 8.8 & 1.01 & 18.4 & 0.55 & 0.07 & 163.4 & 4.9 \\
\hline
\end{tabular}




\section{Apparatus characterization}

\subsection{Turbulent pipe flows}

Results of measured mean and fluctuating streamwise and velocities (TI $\sim 4 \%$ ) at the exit of the turbulent pipe are generally in agreement with those of Laufer [11] as expected and not shown here for brevity. Further, the mean cross-streamwise velocities are essentially 0 and the fluctuating velocities (TI $\sim 4 \%$ ) are approximately uniform for $y / D_{1} \leqslant 0.5$. Isotropy, $u^{\prime} / v^{\prime}$, hence are close to unity for $y / D_{1} \leqslant 0.15$. For flow properties along the axial direction of the pipe, it is found that they are essentially unchanged for $x / D_{1} \leqslant 0.5(60 \mathrm{~mm})$ (not shown). This is reasonable since it is well inside the potential core region of the pipe jet. As for the power spectrum of fluctuating velocities, it will be discussed together with disk flow in the following section. Therefore, a nearly homogeneous and isotropic region $(\sim 4 \% \quad \mathrm{TI})$, $60 \times 30 \times 30 \mathrm{~mm}$, at the exit of the turbulent pipe was successfully constructed.

\subsection{Turbulent pipe flows with a disk plate}

Some typical radial distributions of mean and fluctuating velocities for $U_{\mathrm{c}}=0.6 \mathrm{~m} / \mathrm{s}$ at $L / D_{2}=2.5-25$ for the turbulent pipe flow with a disk plate $\left(D_{2}=20 \mathrm{~mm}\right)$ is presented in Fig. 3. $U_{\mathrm{c}}=0.6 \mathrm{~m} / \mathrm{s}$ was chosen due to the fixed $R e_{d}=100$ required for droplet evaporation experiment described earlier. Similar trends were found for other speeds of the tunnel. In order to maintain constant velocity (e.g., $0.6 \mathrm{~m} / \mathrm{s})$ at the measuring plane $(x=15$ $\mathrm{mm}$ ), blower speed had to be adjusted according to the disk plate location in the pipe. It is found that the streamwise mean and fluctuating velocities are very uniform as $y / D_{1} \leqslant 0.1(12 \mathrm{~mm})$. As shown in Fig. 3, turbulence intensity, $u^{\prime} / U_{\mathrm{c}}$, increases from $5 \%$ to $32 \%$ as $L / D_{2}$ decreases from 25 to 2.5 . In general, the combinations of different disk plate sizes and distances from the tunnel exit provided the flow fields with $u^{\prime} / U_{\mathrm{c}}=5-60 \%$ and $\ell / d=2.5-20$. Isotropy is also found to be near unity for $y / D_{1} \leqslant 0.1$. In terms of mean and fluctuating velocities, we can thus conclude that there existed a nearly isotropic and homogeneous region, $60 \times 24 \times 24 \mathrm{~mm}$, at the tunnel exit even with the disk plate placed in the pipe. However, one last thing we have to check is the power spectrum of the fluctuating velocities, which is described as follows.

Fig. 4 illustrates typical non-dimensional streamwise and cross-streamwise temporal power spectral densities of fluctuating velocities measured for the turbulent pipe flow with $\left(U_{\mathrm{c}}=1 \mathrm{~m} / \mathrm{s}\right)$ and without $\left(U_{\mathrm{c}}=0.5-3 \mathrm{~m} / \mathrm{s}\right)$ a disk plate, which are representative of present test conditions for droplet evaporation experiments. The data were measured near the pipe axis with $x=15 \mathrm{~mm}$. Both the power spectral densities and the frequencies are

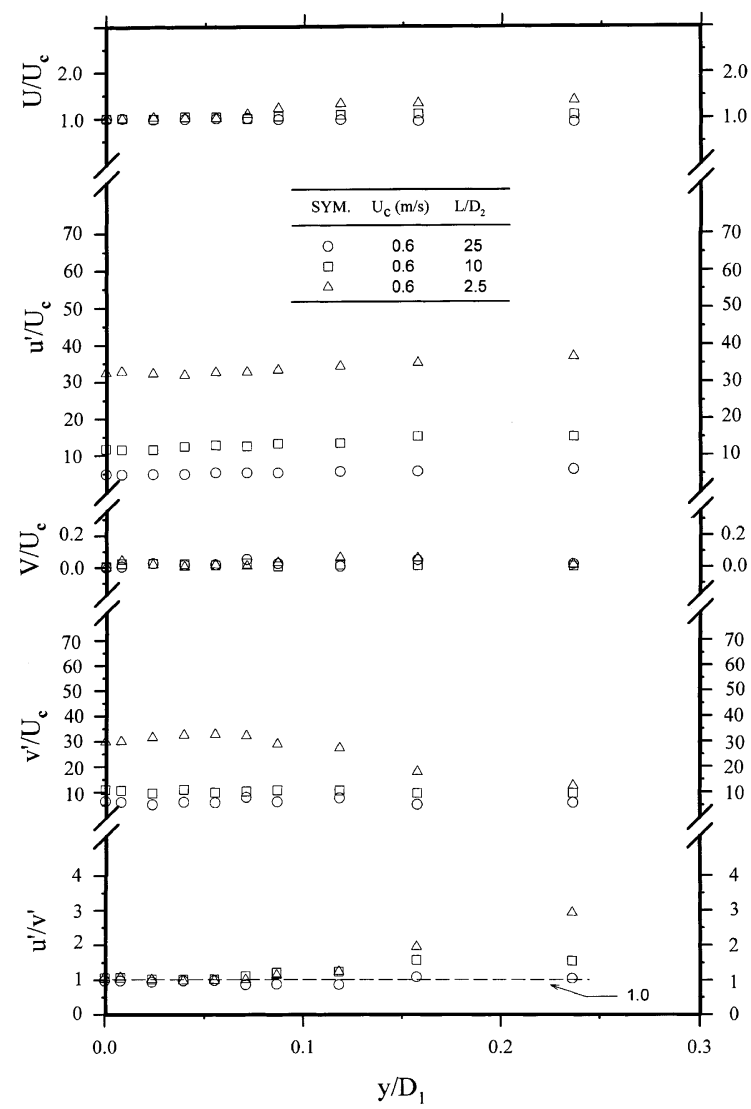

Fig. 3. Radial distribution of mean and fluctuating velocities at the exit of a turbulent pipe with a 20 -mm disk plate.

normalized by the integral timescales, $\tau_{u}$ and $\tau_{v}$, which are pertinent to these flows. $\tau_{u}$ and $\tau_{v}$ are on the order of 30 and $10 \mathrm{~ms}$ for the typical test conditions of droplet evaporation, respectively, but the values vary with the tunnel speed and turbulence intensity. The differences between the measurements without and with a disk plate at different tunnel speeds are small when compared with experimental uncertainties. The step noise caused by the constant sampling period also is comparatively small as can be seen in Fig. 4. For all cases illustrated, there is an obvious hump in the spectrum of cross-streamwise velocity fluctuations in the low-frequency range, which is similar to those observed by Laufer [11], Wu et al. [12], Ruff [13] and Wu and Faeth [10,17]. Moreover, the measurements are also in reasonable agreement with theory of isotropic turbulence, which are represented by the solid lines in Fig. 4. In the high-frequency range, the slopes of the power spectral densities are in the range of $-5 / 3$ and -2 , which means that the flow is close to isotropic or fully developed turbulence.

In summary, the turbulent flow properties at the exit of the tunnel with and without the disk are nearly isotropic and homogeneous $\left(u^{\prime} / U_{\mathrm{c}}=4-60 \%, \ell / d=2.5-20\right)$ 


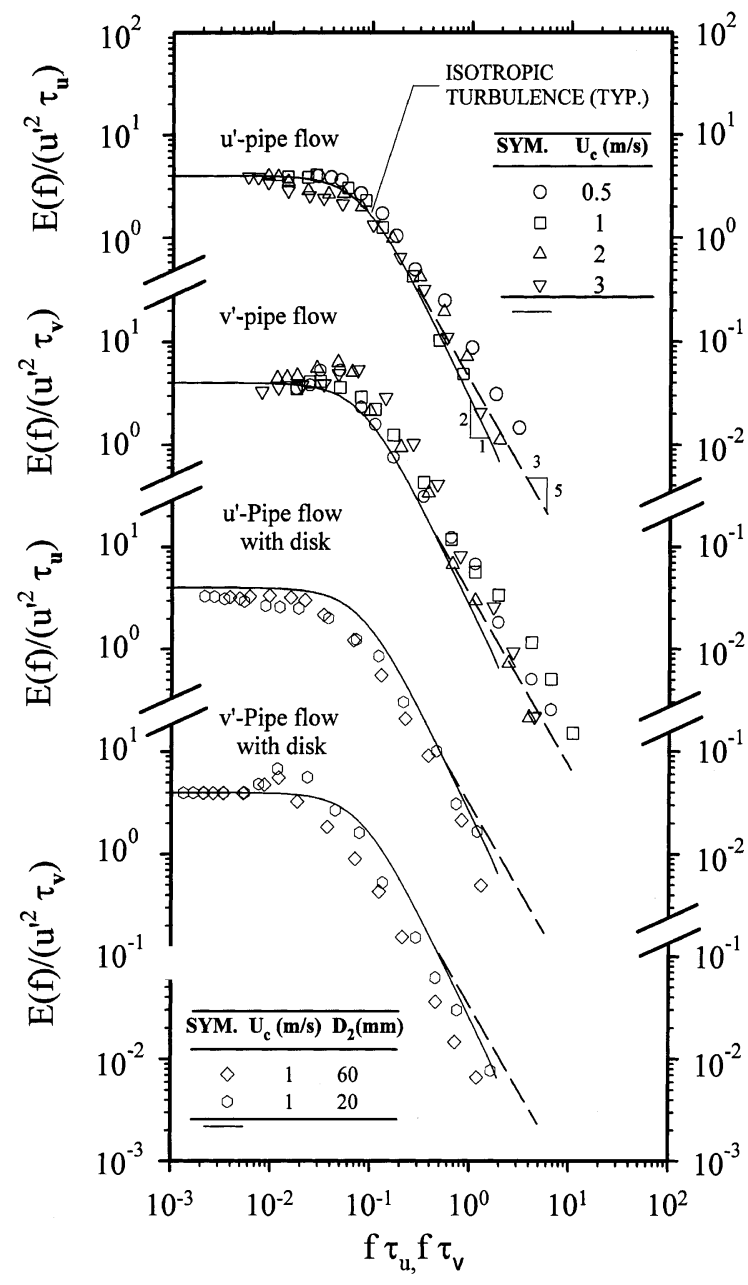

Fig. 4. Temporal power spectrum of streamwise and crossstreamwise velocity fluctuations at the exit of a for turbulent pipe without and with a disk plate $(x=15 \mathrm{~mm})$.

within experimental uncertainties in a region of $24 \times 24 \times 60 \mathrm{~mm}$ near the pipe axis. This region is reasonably uniform for further droplet evaporation measurements discussed later.

\section{Droplet evaporation}

\subsection{Droplet evaporation in quasi-laminar environments}

As mentioned earlier, the quasi-laminar flow conditions were obtained with the contraction nozzle at the end of the wind tunnel, as shown in Fig. 1. Mean and fluctuating streamwise velocities are very uniform at the test section, which are not shown here. The turbulence intensities, $u^{\prime} / U_{\mathrm{c}}$, are about $1 \%$ for $U_{\mathrm{c}}=0.5-3 \mathrm{~m} / \mathrm{s}$. These flow condition were then used as the quasi-lami-

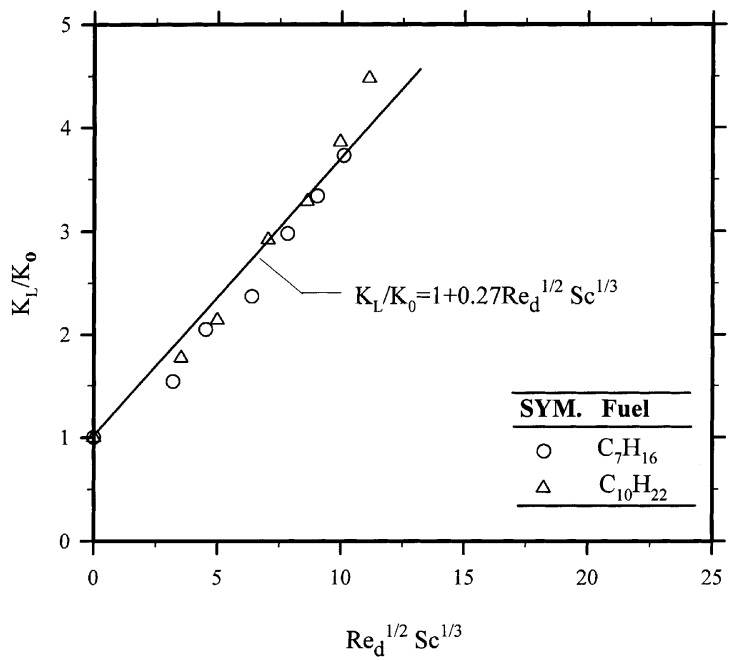

Fig. 5. Measured Frössling correlation for heptane and decane fuel droplets.

nar test condition for droplet evaporation experiments. Fig. 5 illustrates the normalized liquid droplet evaporation rate constant, $K_{\mathrm{L}} / K_{0}$, as a function of $R e_{d}^{1 / 2} S c^{1 / 3}$ at quasi-laminar flow conditions, where $K_{0}$ is the droplet evaporation rate constant at $R e_{d}=0$ (natural convection). It is noted that both $K_{\mathrm{L}}$ and $K_{0}$ are fitted from the normal $d^{2}$-law. Related Frössling correlation [5] can be expressed as

$K_{\mathrm{L}} / K_{0}=1+F_{0} \operatorname{Re}_{d}^{1 / 2} S c^{1 / 3}$,

where $F_{0}$ is the Frössling coefficient and $S c$ is the Schmidt number of the fuel vapor. In the current study, $F_{0}$ is fitted as 0.27 for both fuels, which is close to the value of 0.276 obtained by Frössling [19]. For simplicity of evaluating the experimental data, Schmidt number is defined as

$S c=\frac{\mu_{\mathrm{v}}}{\rho_{\mathrm{v}} D_{\mathrm{AB}}}=\frac{v_{\mathrm{v}}}{D_{\mathrm{AB}}}$,

where dynamic vapor viscosity of the fuel is fitted from [21] and $D_{\mathrm{AB}}$ is found from quasi-steady theory of droplet evaporation using current experimental data at natural convection condition [18]. Not only is the definition simple, but also bears strong physical meaning which represents the ratio of momentum transfer of fuel vapor to mass transfer of fuel vapor in air. Resulting Schmidt number is 0.12 and 0.092 for decane and heptane, respectively. Gokalp et al. [5] have instead obtained 0.18 for Frössling coefficient using $S c=0.71$ assuming unity Lewis number for fuel vapor. Nevertheless, the absolute data of evaporation rate obtained in the current study for quasi-laminar flow condition are compatible with those of Gokalp et al. [5]. 


\subsection{Droplet evaporation in turbulent environments}

In order to further simplify the problem, we have measured the droplet evaporation in turbulent environments at $R e_{d}=100$. There were two sizes of droplet, 1.5 and $2.5 \mathrm{~mm}$, which were used interchangeably to obtain constant $R e_{d}=100$ and suitable length scale ratio $\ell / d$. Fig. 6 shows the typical time history of normalized squared droplet diameter for $n$-decane and $n$-heptane at different turbulence intensities with approximately the same $\ell / d \approx 5$. Data for natural convection at room temperature are also included in Fig. 6. Data are best fitted with $d^{2}$-law as in quasi-laminar environments. At this $\ell / d$, the evaporation rate constant increases with the ambient turbulence intensity for both test fuel droplets. However, the effect is more pronounced as the droplet becomes less volatile, e.g., $n$-decane in this case. Similar trends are observed for other length scale ratios $(\ell / d \approx 2.5,10$ and 20$)$. Indeed, we can conclude that ambient turbulence intensity enhances the evaporation rate from the liquid droplet surface.

Fig. 7 presents the typical time history of normalized squared droplet diameter for $n$-decane and $n$-heptane at different $\ell / d$, but with approximately the same $u^{\prime} / U_{\mathrm{c}} \approx 10 \%$. Droplet evaporation constant generally increases with decreasing $\ell / d$, which represents that the smaller the ambient turbulence length scale relative to the initial droplet size, the faster the liquid evaporates from the droplet surface. Interestingly, $K / K_{0}$ decreases as $\ell / d$ decreases from 7.13 to 3.77 for both fuels. Similar trends are found for other turbulence intensities $\left(u^{\prime} / U_{\mathrm{c}} \approx 4 \%, 15 \%, 30 \%\right.$ and $\left.50 \%\right)$. The results represent that the ratio of the ambient integral length scale to the initial droplet diameter may not be an appropriate

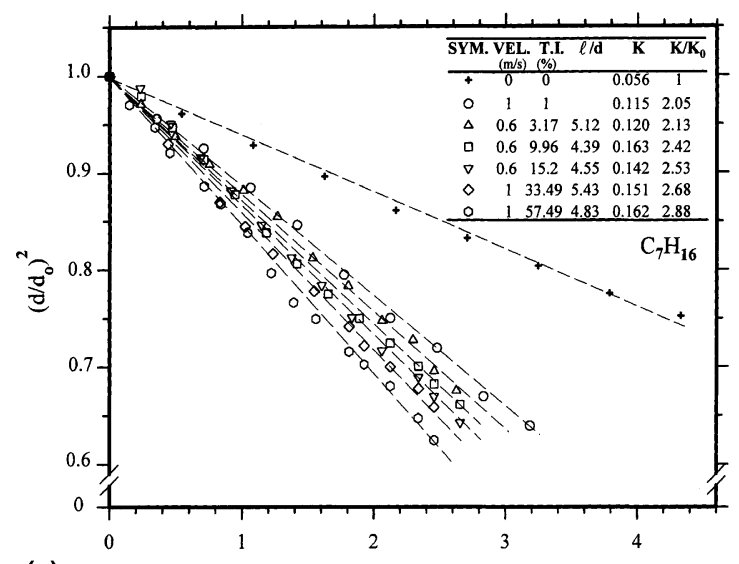

(a)

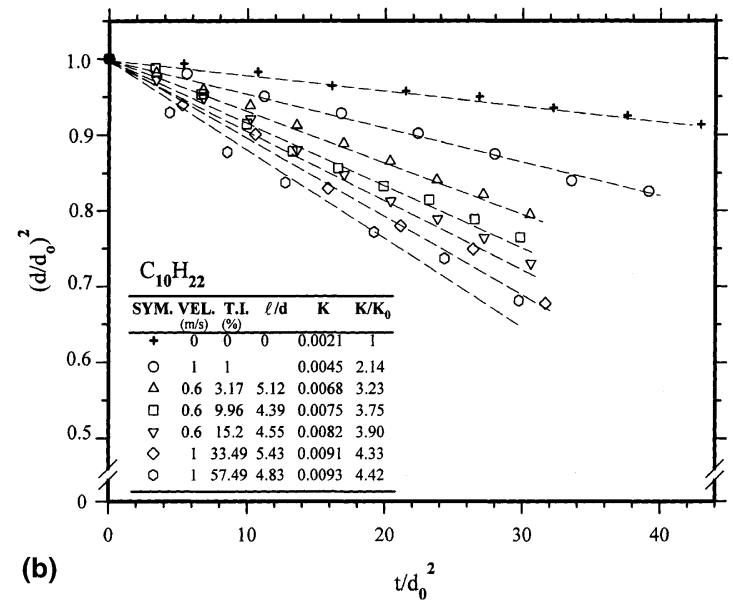

(b)

Fig. 6. Time history of squared normalized droplet diameter at $\ell / d=5$ condition: (a) $\mathrm{C}_{7} \mathrm{H}_{16}$; (b) $\mathrm{C}_{10} \mathrm{H}_{22}$.

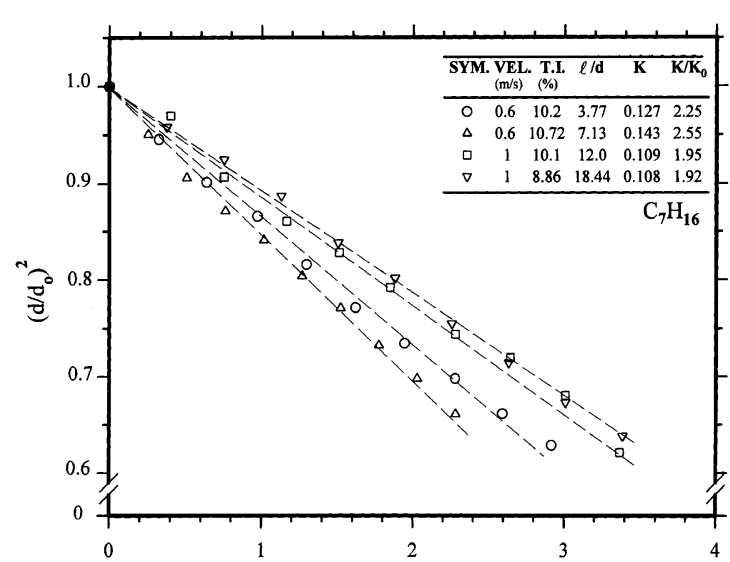

(a)

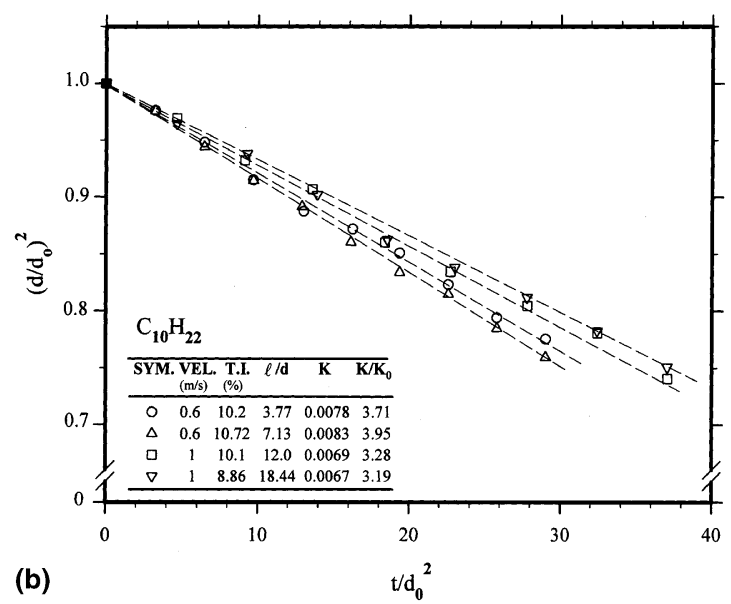

(b)

Fig. 7. Time history of squared normalized droplet diameter at $\mathrm{TI}=10 \%$ condition: (a) $\mathrm{C}_{7} \mathrm{H}_{16}$; (b) $\mathrm{C}_{10} \mathrm{H}_{22}$. 
parameter in characterizing the droplet evaporation in turbulent environments. However, we can conclude that the magnitude of ambient turbulence length scale does play an important role in affecting the droplet evaporation, which has been largely ignored in the past.

Effects of the ambient turbulence intensity on the evaporation rate at different length scale ratios are illustrated in Fig. 8. In the figure, data of Gokalp et al. [5] are also included for comparison. It is clear that not only $K / K_{0}$ of less volatile $n$-decane droplets is generally larger than that of more volatile heptane droplets, but also the increase rates of $K / K_{0}$ with turbulence intensity are similar for both fuels, which contradicts with the results of Gokalp et al. [5]. In [5], they concluded that the increase rate of $K / K_{0}$ with turbulence intensity is larger for less volatile fuel, e.g., decane in this case, as shown as the solid lines in Fig. 8. We will demonstrate this point more clearly later by showing them through the correlation of Damköhler number. The data obtained by Gokalp et al. [5], which did not specify the turbulence length scale effects in their report by only stating that $\ell / d>5$, were

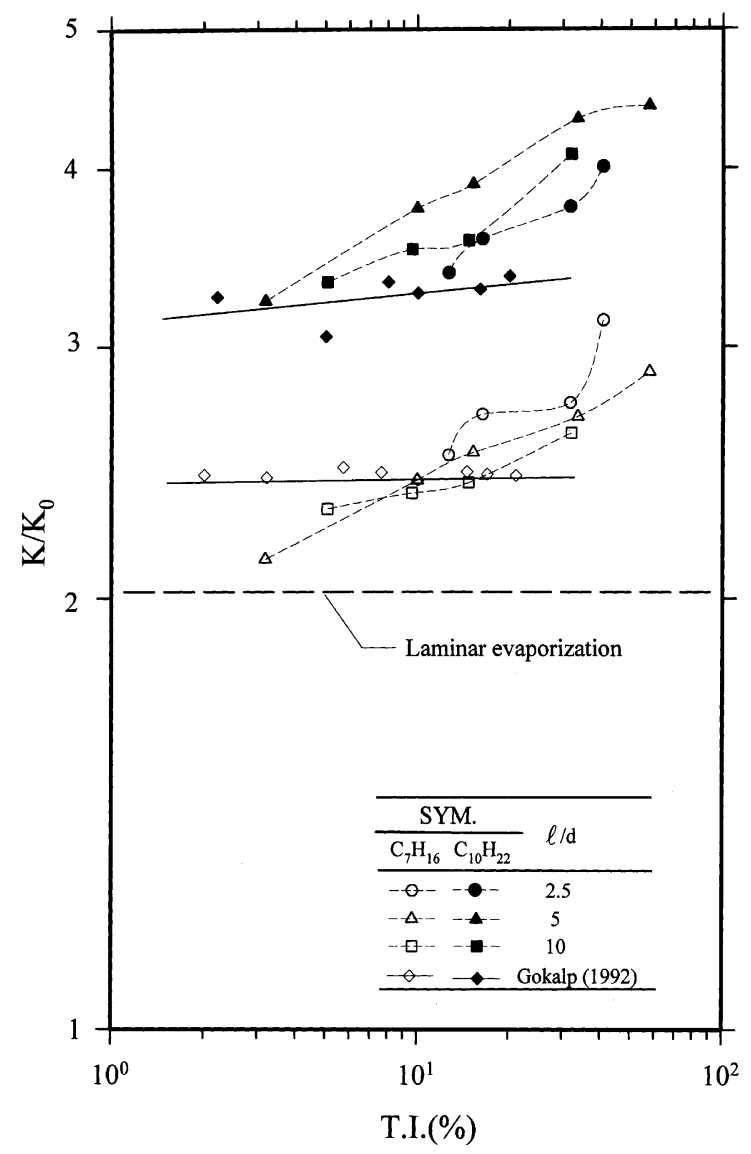

Fig. 8. Variations of normalized vaporization rate with turbulence intensity for heptane and decane fuel droplets. on the same order of the current results without any doubt.

The results have shown that both the turbulence integral length scale and turbulence intensity are not well enough to solely characterize the droplet evaporation in turbulent environments. In contrast, Gokalp et al. [5] has proposed an effective vaporization Damköhler number to roughly explain the effects of the ambient turbulence on the liquid droplet evaporation at room temperature. However, no systematic study of the vaporization Damköhler number on the droplet evaporation has been conducted since then. Therefore, it is interesting to reevaluate this parameter using the current measurements of droplet evaporation. Definition of vaporization Damköhler number [5] is briefly described as follows for completeness:

$D a_{\mathrm{v}}=\frac{T_{\mathrm{ed}}}{T_{\mathrm{v}}}$,

$T_{\text {ed }}=\frac{\left(d^{3} \ell\right)^{1 / 3}}{u^{\prime}}=\frac{d^{2 / 3}}{\varepsilon^{1 / 3}}$,

$\varepsilon=A \frac{u^{\prime 3}}{\ell}$

$T_{\mathrm{v}}=\delta_{\mathrm{M}} / V_{\mathrm{r}}$,

where $A=1$ is used in the current analysis, and $\delta_{\mathrm{M}}$ (vapor film thickness) and $V_{\mathrm{r}}$ (vapor blowing velocity) are computed by using the theory developed by Abramzon and Sirignano [20]. The basic assumption in defining $T_{\text {ed }}$ is that inertial subrange exists between the integral length scale and the Komogorov scale, and the droplet size lies in this range, which is compatible with the current test conditions (see Tables 1 and 2). For demonstration, a calculation example of $D a_{\mathrm{v}}$ for decane and heptane (TI $=10 \%, d_{\mathrm{p}}=1.5 \mathrm{~mm}, U=1 \mathrm{~m} / \mathrm{s}, \ell=18$ $\mathrm{mm}$ ) is shown in Table 3. All the formulas used to compute the parameters are adopted from those of Abramzon and Sirignano [20] and are listed in the 'Nomenclature' for reference. Note that physical parameters used in the calculation are based on room temperature other than $1 / 3$ rule that often used in hightemperature environment [22]. This is justified due to the fact that surface temperature is close to room temperature in the current study.

Fig. 9 illustrates the normalized evaporation rate constant $\left(K / K_{\mathrm{L}}\right)$ as a function of the Damköhler number $\left(D a_{\mathrm{v}}\right)$, which extends over three decades. Surprisingly from the current results, $K / K_{\mathrm{L}}$ correlates reasonably well with $D a_{\mathrm{v}}$ and decreases with $D a_{\mathrm{v}}$. For small Damköhler number cases $\left(D a_{\mathrm{v}}<0.002\right)$, the increase of droplet evaporation rate due to the ambient turbulence can be as high as 100\%, as shown in Fig. 9. However, for very large Damköhler number cases $\left(D a_{\mathrm{v}}>0.1\right)$, the effect of the ambient turbulence is diminishing to be negligible. Physically, as the Damköhler 

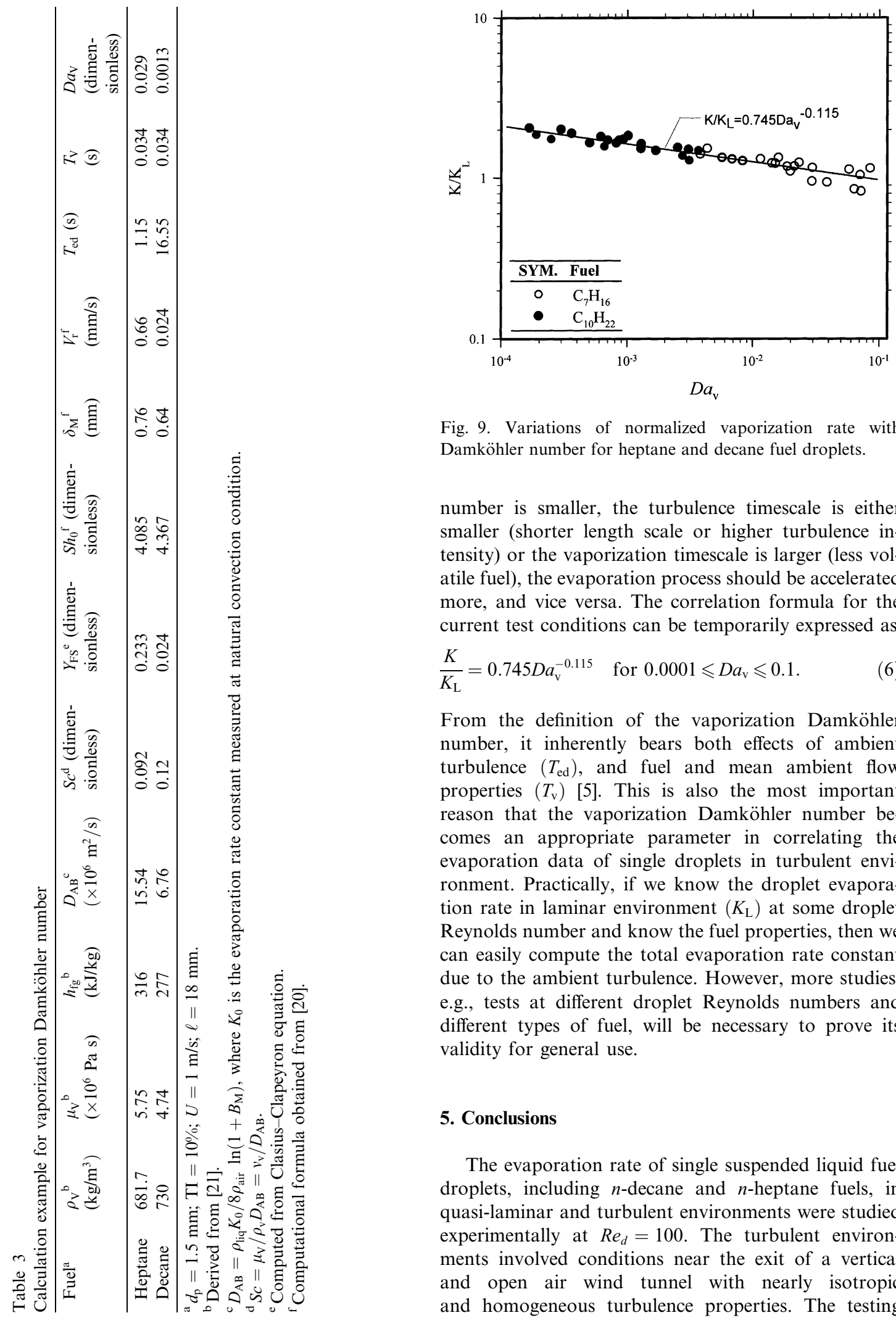

Fig. 9. Variations of normalized vaporization rate with Damköhler number for heptane and decane fuel droplets.

number is smaller, the turbulence timescale is either smaller (shorter length scale or higher turbulence intensity) or the vaporization timescale is larger (less volatile fuel), the evaporation process should be accelerated more, and vice versa. The correlation formula for the current test conditions can be temporarily expressed as

$\frac{K}{K_{\mathrm{L}}}=0.745 D a_{\mathrm{v}}^{-0.115}$ for $0.0001 \leqslant D a_{\mathrm{v}} \leqslant 0.1$.

From the definition of the vaporization Damköhler number, it inherently bears both effects of ambient turbulence $\left(T_{\text {ed }}\right)$, and fuel and mean ambient flow properties $\left(T_{\mathrm{v}}\right)$ [5]. This is also the most important reason that the vaporization Damköhler number becomes an appropriate parameter in correlating the evaporation data of single droplets in turbulent environment. Practically, if we know the droplet evaporation rate in laminar environment $\left(K_{\mathrm{L}}\right)$ at some droplet Reynolds number and know the fuel properties, then we can easily compute the total evaporation rate constant due to the ambient turbulence. However, more studies, e.g., tests at different droplet Reynolds numbers and different types of fuel, will be necessary to prove its validity for general use.

\section{Conclusions}

The evaporation rate of single suspended liquid fuel droplets, including $n$-decane and $n$-heptane fuels, in quasi-laminar and turbulent environments were studied experimentally at $R e_{d}=100$. The turbulent environments involved conditions near the exit of a vertical and open air wind tunnel with nearly isotropic and homogeneous turbulence properties. The testing 
turbulent flow conditions include turbulence intensities of roughly $1-60 \%, \ell / d$ in the range $2.5-20$, and $\eta / d$ in the range $0.02-0.13$. The major findings of study are as follows:

(a) A nearly isotropic and homogeneous turbulent test region was successfully constructed with the mean streamwise flow velocity in the range $0.5-3 \mathrm{~m} / \mathrm{s}$, which are well suited for studying ambient turbulence effects on droplet evaporation.

(b) Present measurements of droplet evaporation rate in quasi-laminar and turbulent environments agree basically with those of Frössling [19] and Gokalp et al. [5], respectively.

(c) Combined effects of the ambient turbulence and the fuel properties on the droplet evaporation rate at room temperature can be reasonably correlated with the vaporization Damköhler number proposed by Gokalp et al. [5]. In general, the measured normalized droplet evaporation rate, $K / K_{\mathrm{L}}$, decreases as the Damköhler number, $D a_{\mathrm{v}}$, increases. Correlated results show that $K / K_{\mathrm{L}}$ is proportional to $D a_{\mathrm{v}}^{-0.115}$ over the present testing range at room temperature. Furthermore, the ambient turbulence will not accelerate the vaporization process for Damköhler number larger than 0.1.

Effects of droplet Reynolds number, fuel type (volatility) and ambient temperature (higher temperature) must still be resolved in order to construct a pertinent model for droplet vaporization in a practical spray system. Moreover, a broader range of $\ell / d$ (e.g., smaller $\ell / d$ ) should be investigated in order to better understand the interaction between ambient turbulence length scales and droplet evaporation process. Work in this direction is currently in progress.

\section{Acknowledgements}

The authors would like to express their sincere thanks to the financial support by the National Science Council of Taiwan under Grants NSC-88-2212-E-009019 and NSC-89-2212-E-009-034.

\section{References}

[1] G.M. Faeth, Evaporation and combustion of sprays, Progr. Energy Combust. Sci. 9 (1983) 1-76.

[2] W.A. Sirignano, Fuel droplet vaporation and spray combustion theory, Progr. Energy Combust. Sci. 9 (1983) 291322.

[3] C.M. Megaridis, W.A. Siriginano, in: ASME Winter Meeting, Dallas, TX, 1990.
[4] J.M. Tishkoff, Interaction between droplets and gas-phase turbulent flows, in: Third Joint ASCE/ASME Mechanics conference, 1989, pp. 1-6.

[5] I. Gokalp, C. Chauveau, O. Simon, X. Chesneau, Mass transfer from liquid fuel droplets in turbulent flow, Combust. Flame 89 (1992) 286-298.

[6] N. Hiromitsu, O. Kawaguchi, Influence of flow turbulence on the evaporation rate of a suspended droplet in a hot air flow, Heat Transfer - Japan. Res. 24 (1997) 689-700.

[7] P.R.A. Yearling, Experimental determination of convective heat and mass transfer rates from single evaporating liquid droplets in a turbulent air flow, Ph.D. Thesis, North Carolina State University, Raleigh, NC, USA, 1995.

[8] M. Birouk, C. Chauveau, B. Sarh, A. Quilgars, I. Gokalp, Turbulence effects on the vaporization of monocomponent single droplets, Combust. Sci. Technol. 113-114 (1996) 413-428.

[9] I. Gokalp, C. Chauveau, B. Vieille, C. Morin, M. Birouk, B. Gelfand, New models for droplet break-up and vaporization for high pressure spray combustion applications, in: 12th PERC Symposium, Pennsylvania State University, State College, November 18 and 19, 1999.

[10] J.-S Wu, G.M. Faeth, Effect of ambient turbulence intensity on sphere wakes at intermediate Reynolds numbers, AIAA J. 33 (1995) 171-173.

[11] J. Laufer, The structure of turbulence in fully developed pipe flow, NACA Report 1174, August, 1954.

[12] M.-S. Wu, S. Kwon, J.F. Driscoll, G.M. Faeth, Preferential diffusion effects on the surface structure of turbulent premixed hydrogen/air flame, Combust. Sci. Technol. 78 (1991) 69-96.

[13] G.A. Ruff, Structure and mixing properties of the nearinjector region of nonevaporating pressure-atomized sprays, Ph.D. Thesis, University of Michigan, Ann Arbor, MI, 1990, pp. 54-58.

[14] J.O. Hinze, Turbulence, second ed., McGraw-Hill, New York, 1975.

[15] G.M. Faeth, Mixing, transport and combustion in sprays, Progr. Energy Combust. Sci. 13 (1987) 293-345.

[16] H. Tennekes, J.L. Lumley, A First Course in Turbulence, MIT Press, Cambridge, MA, 1972, pp. 113-124.

[17] J.-S. Wu, G.M. Faeth, Sphere wakes at moderate Reynolds numbers in a turbulent environment, AIAA J. 32 (1994) 535-541.

[18] S.R. Turns, An Introduction to Combustion, McGrawHill, New York, 1996.

[19] N. Frössling, Gerlands Beitr. Geophys. 52 (1938) 170-175.

[20] B. Abramzon, W.A. Sirignano, Droplet vaporization model for spray combustion calculations, Int. J. Heat Mass Transfer 32 (1989) 1605-1618.

[21] T.E. Daubert, R.P. Danner, Physical and thermodynamic properties of pure chemicals: data compilation, Hemisphere, Washington, DC, 1989.

[22] M. Renksizbulut, M.C. Yuen, Experimental study of droplet evaporation in a high-temperature air stream, J. Heat Transfer 105 (1982) 384-388. 